\title{
Interpolated Coarse Models for Microwave Design Optimization With Space Mapping
}

\author{
Slawomir Koziel, Senior Member, IEEE, and John W. Bandler, Life Fellow, IEEE
}

\begin{abstract}
The efficiency of space-mapping optimization depends on the quality of the underlying coarse model, which should be sufficiently close to the fine model and cheap to evaluate. In practice, available coarse models are often cheap, but inaccurate (e.g., a circuit equivalent of the microwave structure) or accurate, but too expensive (e.g., a coarse-mesh model). In either case, the space-mapping optimization process exhibits substantial computational overhead due to the excessive fine model evaluations necessary to find a good solution if the coarse model is inaccurate, or due to the cost of the parameter extraction and surrogate optimization sub-problems if the coarse model is too expensive. In this paper, we use an interpolation technique, which allows us to create coarse models that are both accurate and cheap. This overcomes the accuracy/cost dilemma described above, permitting significant reduction of the space-mapping optimization time. Examples verify the performance of our approach.
\end{abstract}

Index Terms-Coarse model, engineering optimization, microwave design, space mapping, space-mapping optimization.

\section{INTRODUCTION}

$\mathbf{S}$ PACE MAPPING [1]-[5] is a methodology that allows efficient optimization of expensive or "fine" models by means of the iterative optimization and updating of so-called "coarse" models, which are less accurate, but cheaper to evaluate. Provided that the misalignment between the fine and coarse models is not significant, space-mapping-based algorithms typically provide excellent results after only a few evaluations of the fine model. A similar idea is shared by other surrogate-model-based methods [6]-[12], however, many of them do not use a coarse model: the surrogate model is created by direct approximation of the available fine model data.

Space mapping is widely used in the optimization of microwave devices [1]-[3], [13]-[17], where fine models are often based on full-wave electromagnetic simulations, whereas coarse models may be physically based circuit models. Recently, space-mapping techniques have been applied to design problems in a growing number of areas (e.g., [18]-[20]).

Manuscript received February 2, 2007. This work was supported in part by the Natural Sciences and Engineering Research Council of Canada under Grant OGP0007239 and Grant STPGP336760, and by Bandler Corporation.

S. Koziel is with the Simulation Optimization Systems Research Laboratory, Department of Electrical and Computer Engineering, McMaster University, Hamilton, ON, Canada L8S 4K1 (e-mail: koziels@ mcmaster.ca).

J. W. Bandler is with the Simulation Optimization Systems Research Laboratory, Department of Electrical and Computer Engineering, McMaster University, Hamilton, ON, Canada L8S 4K1, and also with Bandler Corporation, Dundas, ON, Canada L9H 5E7 (e-mail: bandler@mcmaster.ca).

Color versions of one or more of the figures in this paper are available online at http://ieeexplore.ieee.org.

Digital Object Identifier 10.1109/TMTT.2007.902618
A number of papers cover different aspects of space mapping, including the development of new algorithms ([2], [3], [21], [22]), space-mapping-based modeling [23]-[28], theoretical foundations ([21], [29], [30]), etc.

It is well known that the performance of a space-mapping optimization algorithm depends on the quality of the underlying coarse model, which should be as good a representation of the fine model to be optimized as possible. On the other hand, the coarse model should also be easy to optimize and significantly less expensive than the fine model. Under these conditions, a space-mapping algorithm can reach a satisfactory solution after a few fine model evaluations. Moreover, the total cost related to the parameter extraction and surrogate optimization sub-problems, involving multiple coarse model evaluations, is negligible in comparison with the total cost of fine model evaluation.

In practice, however, available coarse models are either cheap, but inaccurate, e.g., a circuit equivalent of the microwave structure, or accurate, but too expensive, e.g., a microwave structure evaluated using the same simulator as the fine model, but with a coarser mesh. In the first case, the space-mapping optimization process exhibits substantial computational overhead due to the excessive fine model evaluations necessary to find a good solution (i.e., the number of space-mapping iterations is larger than it could be if the accurate model were used). In the latter case, the total cost of solving the parameter extraction and surrogate optimization sub-problems may be comparable with the total cost of fine model evaluation or may even determine the total cost of the space-mapping optimization process.

In this paper, we utilize an interpolation technique, which allows us to create coarse models that are both accurate and, at the same time, sufficiently cheap. In particular, the original coarse model is evaluated on a relatively coarse simulation grid and the modified model is obtained by interpolating this data using a suitable methodology. In this way, the original coarse model (which is typically assumed to exhibit sufficient accuracy, but is too expensive to make space-mapping optimization efficient) is evaluated at a limited number of points, which allows us to reduce the total space-mapping optimization time.

\section{Motivation}

Let us consider the following optimization problem: a second-order tapped-line microstrip filter [31] shown in Fig. 1. The design parameters are $\boldsymbol{x}=\left[L_{1} g\right]^{T}$. The fine model $\boldsymbol{R}_{f}$ is simulated in FEKO [32]. The number of meshes for the fine model is 360 , which ensures mesh convergence for the 


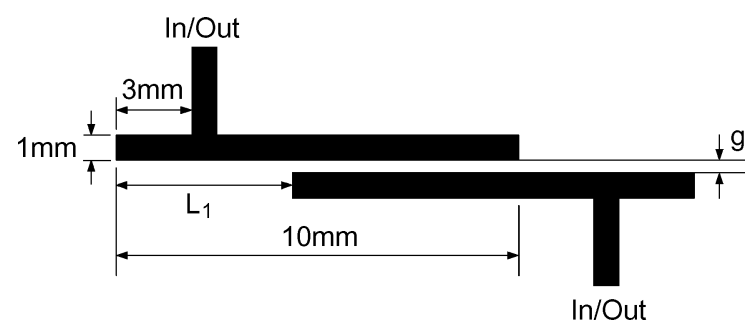

Fig. 1. Geometry of the second-order tapped-line microstrip filter [31].

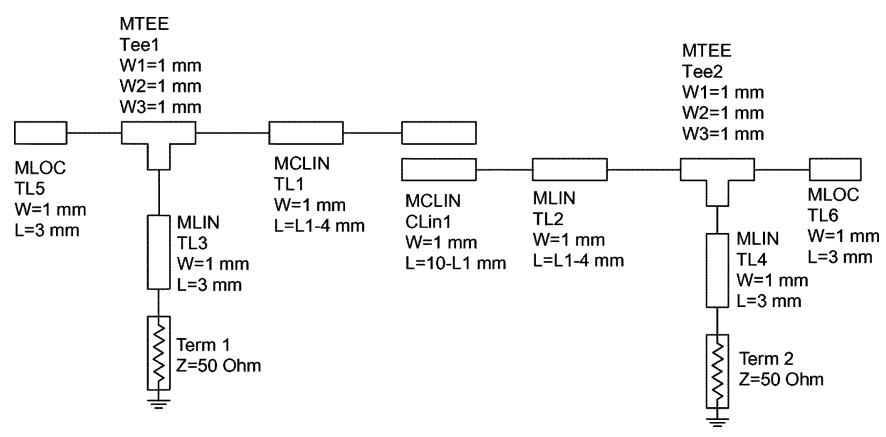

Fig. 2. Coarse model $\boldsymbol{R}_{c 2}$ of the second-order tapped-line filter (Agilent ADS).

structure. Simulation time for the fine model is $290 \mathrm{~s}$. The design specifications are

$$
\begin{aligned}
& \left|S_{21}\right| \leq-20 \mathrm{~dB} \text { for } 3.0 \mathrm{GHz} \leq \omega \leq 4.0 \mathrm{GHz} \\
& \left|S_{21}\right| \geq-3 \mathrm{~dB} \text { for } 4.75 \mathrm{GHz} \leq \omega \leq 5.25 \mathrm{GHz} \\
& \left|S_{21}\right| \leq-20 \mathrm{~dB} \text { for } 6.0 \mathrm{GHz} \leq \omega \leq 7.0 \mathrm{GHz} .
\end{aligned}
$$

We also use $\boldsymbol{R}_{f}$ to denote the response vector of the fine model. In this case, the model response is the evaluation of $\left|S_{21}\right|$ at 33 frequency points uniformly distributed in the interval from 3 to $7 \mathrm{GHz}$.

We consider two coarse models. Model $\boldsymbol{R}_{c 1}$ is the structure in Fig. 1, also simulated in FEKO; however, the number of meshes is only 48 . The number of meshes for $\boldsymbol{R}_{f}$ and $\boldsymbol{R}_{c 1}$ are measured at the optimal solution of $\boldsymbol{R}_{c 1}$, which is $\boldsymbol{x}_{1}^{(0)}=$ $\left[\begin{array}{ll}5.071 & 0.0404\end{array}\right]^{T} \mathrm{~mm}$. The simulation time for $\boldsymbol{R}_{c 1}$ is approximately $11 \mathrm{~s}$. Model $\boldsymbol{R}_{c 2}$ is the circuit model implemented in Agilent ADS [33] shown in Fig. 2. Evaluation time for $\boldsymbol{R}_{c 2}$ is approximately $1.2 \mathrm{~s}$. As before, symbols $\boldsymbol{R}_{c 1}$ and $\boldsymbol{R}_{c 2}$ are also used to denote the response vectors of the respective models.

For this problem, we used input space mapping and output space mapping [21]. In particular, the space-mapping surrogate model is defined as $\boldsymbol{R}_{s}(\boldsymbol{x})=\boldsymbol{R}_{c}(\boldsymbol{x}+\boldsymbol{c})+\boldsymbol{d}$, where vector $\boldsymbol{c}$ is found using parameter extraction [21], after which $\boldsymbol{d}$ is the residual vector evaluated by $\boldsymbol{d}=\boldsymbol{R}_{f}(\boldsymbol{x})-\boldsymbol{R}_{c}(\boldsymbol{x}+\boldsymbol{c})$. We perform space-mapping optimization twice: using model $\boldsymbol{R}_{c 1}$ with its optimal solution $\boldsymbol{x}_{1}^{(0)}$ as a starting point, and then using model $\boldsymbol{R}_{c 2}$ with its corresponding optimal solution $\boldsymbol{x}_{2}^{(0)}=$ $\left[\begin{array}{ll}6.977 & 0.060\end{array}\right]^{T} \mathrm{~mm}$ as a starting point.

Figs. 3 and 4 show the responses of the fine and coarse model $\boldsymbol{R}_{c 1}$ at $\boldsymbol{x}_{1}^{(0)}$, as well as the fine and coarse model $\boldsymbol{R}_{c 2}$ at $\boldsymbol{x}_{2}^{(0)}$, respectively. It is seen that the model $\boldsymbol{R}_{c 1}$ exhibits better accuracy

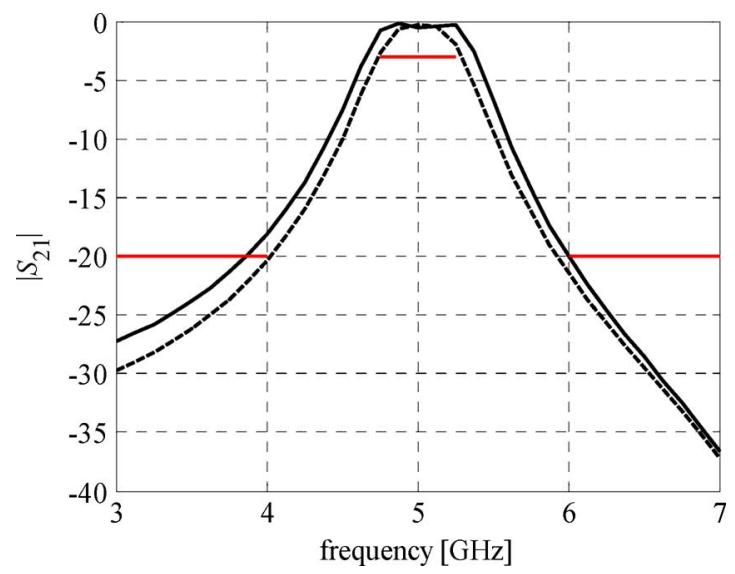

Fig. 3. Second-order tapped-line filter: initial fine model response (solid line) and coarse model $\boldsymbol{R}_{c 1}$ response (dashed line) at $\boldsymbol{x}_{1}^{(0)}$.

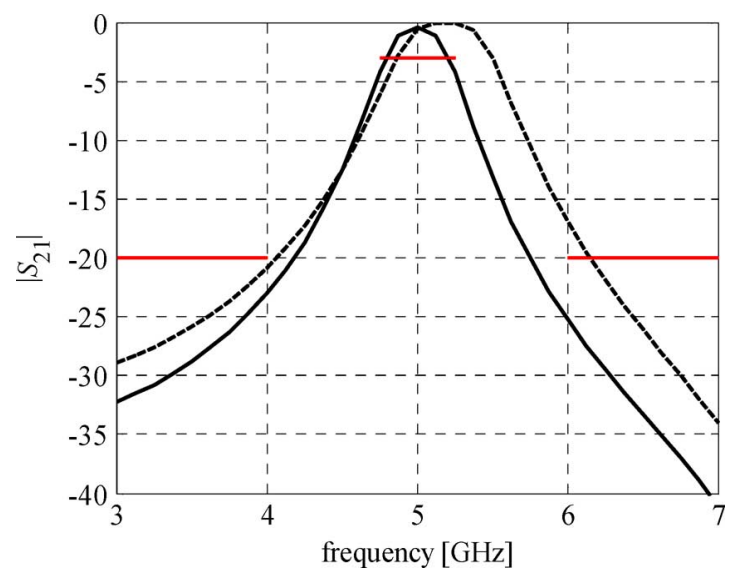

Fig. 4. Second-order tapped-line filter: initial fine model response (solid line) and coarse model $\boldsymbol{R}_{c 2}$ response (dashed line) at $\boldsymbol{x}_{2}^{(0)}$.

than the model $\boldsymbol{R}_{c 2}$ with respect to matching the fine model response. Note also that the $\boldsymbol{R}_{c 2}$ response at its optimal solution does not satisfy the design specifications.

Table I shows the optimization results. The optimized fine model responses are shown in Fig. 5. As we can see, the final specification error is almost independent of which a coarse model is used in the space-mapping algorithm. Different responses reflect different optima found by the algorithm in both cases: [3.897 0.145$]^{T}$ for $\boldsymbol{R}_{c 1}$ and $\left[\begin{array}{lll}5.989 & 0.060\end{array}\right]^{T}$ for $\boldsymbol{R}_{c 2}$. However, because model $\boldsymbol{R}_{c 1}$ is more accurate than $\boldsymbol{R}_{c 2}$, the optimization result is obtained with a smaller number of fine model evaluations. On the other hand, because $\boldsymbol{R}_{c 2}$ is much cheaper to evaluate than $\boldsymbol{R}_{c 1}$, the relative computational cost of solving the parameter extraction and surrogate model optimization sub-problems is much higher for the algorithm using $\boldsymbol{R}_{c 1}$ than for the algorithm using $\boldsymbol{R}_{c 2}(59 \%$ versus $10 \%)$. Hence, the total optimization time is larger for $\boldsymbol{R}_{c 1}$ than for $\boldsymbol{R}_{c 2}$.

It should also be mentioned that model $\boldsymbol{R}_{c 1}$ does not need to achieve mesh convergence because it is a coarse model. However, as an effect of the lack of mesh convergence, the mesh topology and number of mesh elements vary due to the variation of geometrical design parameters during optimization. Consequently, model $\boldsymbol{R}_{c 1}$ is more difficult to optimize than $\boldsymbol{R}_{c 2}$, which 
TABLE I

SPACE-MAPPING OPTIMIZATION RESULTS FOR THE SECOND-ORDER TAPPED-LINE MICROSTRIP FILTER

\begin{tabular}{|c|c|c|c|c|c|}
\hline $\begin{array}{l}\text { Coarse } \\
\text { Model }\end{array}$ & $\begin{array}{l}\text { Final } \\
\text { Spec. } \\
\text { Error } \\
{[\mathrm{dB}]} \\
\end{array}$ & $\begin{array}{l}\text { Number of } \\
\text { Fine Model } \\
\text { Evaluations* }\end{array}$ & $\begin{array}{c}\text { Total } \\
\text { Optimization } \\
\text { Time }\end{array}$ & $\begin{array}{c}\text { Total } \\
\text { Fine Model } \\
\text { Evaluation } \\
\text { Time } \\
\end{array}$ & $\begin{array}{c}\text { Total Parameter } \\
\text { Extraction } \\
\text { and Surrogate } \\
\text { Optimization Time }\end{array}$ \\
\hline & -0.64 & & & $145 \mathrm{~min}(410 \%$ & $(59 \%)$ \\
\hline & -0.65 & 4 & $25.2 \mathrm{~min}$ & $22.6 \min (90 \%)$ & $2.6 \min (10 \%)$ \\
\hline
\end{tabular}

* Includes fine model evaluation at the starting point

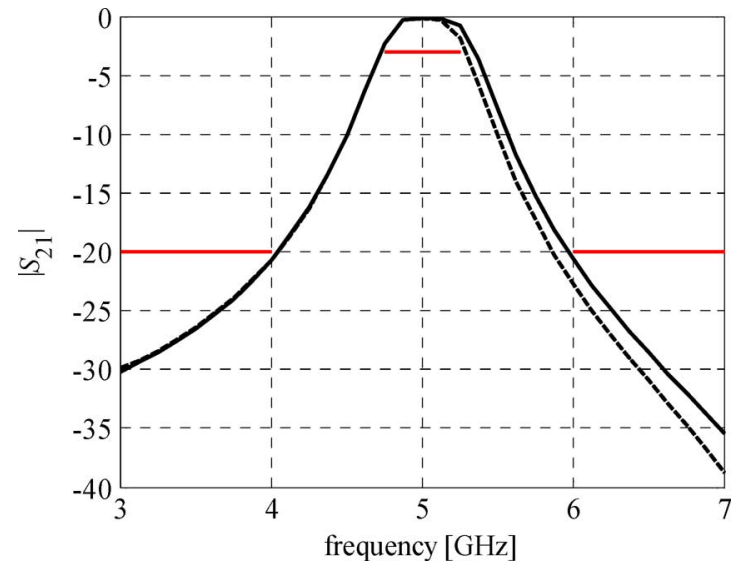

Fig. 5. Second-order tapped-line filter: final fine model response at the solution obtained with space mapping using the $\boldsymbol{R}_{c 1}$ model (solid line) and the $\boldsymbol{R}_{c 2}$ model (dashed line).

is reflected in a larger number of evaluations while performing parameter extraction and surrogate optimization.

As mentioned earlier, model $\boldsymbol{R}_{c 1}$ is accurate, but expensive (and not easy to optimize), while model $\boldsymbol{R}_{c 2}$ is cheap, but not accurate. It can be inferred from the data in Table I that the cost of space-mapping optimization can be substantially reduced if we can provide a coarse model that is accurate, cheap, and easy to optimize. Section III introduces the concept that satisfies these conditions.

\section{INTERPOLATED COARSE MODELS}

\section{A. Notation and Concept}

Let $\boldsymbol{R}_{c}: X \rightarrow R^{m}, X \subseteq R^{n}$ be an original coarse model, which will typically be the model evaluated by the same simulator as the fine model, but using a coarse mesh (as in the example of Section II). Let $G$ be a grid $G(\boldsymbol{\lambda})=\left\{\left[\begin{array}{lll}z_{1} \lambda_{1} & z_{2} \lambda_{2} \ldots z_{n} \lambda_{n}\end{array}\right]^{T}: z_{i} \in Z, i=1, \ldots, n\right\}$,

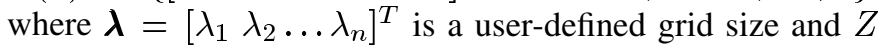
denotes the set of integers; $n$ is the number of design variables. Grid $G$ divides $R^{n}$ into hypercubes with points $\boldsymbol{y} \in G(\boldsymbol{\lambda})$ being corners of these hypercubes. For each $\boldsymbol{y} \in G(\boldsymbol{\lambda})$, we define $c(\boldsymbol{y})=\left[\begin{array}{llll}y_{1}+\lambda_{1} / 2 & y_{2}+\lambda_{2} / 2 & \ldots & y_{n}+\lambda_{n} / 2\end{array}\right]^{T}$ as the center of the corresponding hypercube, and denote by $H(\boldsymbol{y})$ the hypercube itself. Fig. 6 shows an example of the grid and hypercubes for $n=2$.

With each $\boldsymbol{y} \in G(\boldsymbol{\lambda})$, we associate a base set $X_{B}(\boldsymbol{y})$, which is a set of points located in the hypercube with center $c(\boldsymbol{y})$. We will denote by $R_{B}(y)$ a set of responses of the original coarse model $\boldsymbol{R}_{c}$ evaluated at points from $X_{B}(\boldsymbol{y})$.

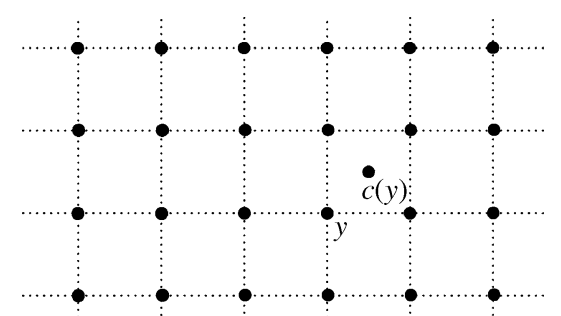

Fig. 6. Grid example for the 2-D case.

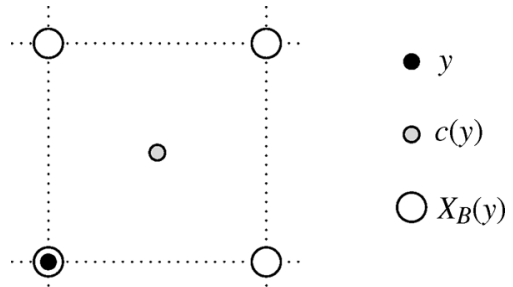

Fig. 7. Example of the base set for fuzzy-system interpolation $n=2$.

Let $F\left(\cdot, X_{B}, R_{B}\right)$ be the function interpolating the data pairs $\left(X_{B}, R_{B}\right) . F(\boldsymbol{x})=F\left(\boldsymbol{x}, X_{B}, R_{B}\right)$ denotes the value of the function $F$ at point $x$.

For each $\boldsymbol{x} \in R^{n}$, we define $s(\boldsymbol{x}) \in G(\boldsymbol{\lambda})$ as $s(\boldsymbol{x})=\left[\lambda_{1}\right.$. $\left.\left\lfloor x_{1} / \lambda_{1}\right\rfloor \lambda_{2} \cdot\left\lfloor x_{2} / \lambda_{2}\right\rfloor \ldots \lambda_{n} \cdot\left\lfloor x_{n} / \lambda_{n}\right\rfloor\right]^{T}$. In other words, $s(\boldsymbol{x})$ is the result of "rounding" $\boldsymbol{x}$ to one of the grid points.

We define an interpolated coarse model $\overline{\boldsymbol{R}}_{c}$ as follows:

$$
\overline{\boldsymbol{R}}_{c}(\boldsymbol{x})=F\left(\boldsymbol{x}, X_{B}(s(\boldsymbol{x})), R_{B}(s(\boldsymbol{x}))\right) .
$$

In the remaining discussion here, we consider the realization of this concept, as well as implementation details. We employ fuzzy systems, techniques successfully used in the computeraided design of microwave structures by other authors (e.g., [34] and [35]).

\section{B. Realization}

It is desirable that the model $\overline{\boldsymbol{R}}_{c}$ is a continuous function, as this will facilitate further optimization of the space-mapping surrogate. This can be achieved using a fuzzy-system interpolation based on the points located at the corners of the hypercubes defined by the grid $G$. In particular, we have $X_{B}(\boldsymbol{y})=\{\boldsymbol{x}$ : $\boldsymbol{x}=\left[\begin{array}{lll}y_{1}+e_{1} \lambda_{1} & y_{2}+e_{2} \lambda_{2} & y_{n}+e_{n} \lambda_{n}\end{array}\right]^{T}, e_{i} \in\{0,1\}, I=$ $1,2, \ldots, n\}$. An example of the base set for $n=2$ is shown in Fig. 7. Note that the number of base points is $N=2^{n}$.

In this study, we use a fuzzy system with triangle membership functions and centroid defuzzification [36]. The fuzzy system uses data pairs $\left(\boldsymbol{x}^{k}, \boldsymbol{R}^{k}\right)$, where $\boldsymbol{x}^{k} \in X_{B}(\boldsymbol{y})$ and $\boldsymbol{R}^{k}=\boldsymbol{R}_{c}\left(\boldsymbol{x}^{k}\right), k=1,2, \ldots, N$. In our realization, each interval $\left[y_{i}, y_{i}+\lambda_{i}\right], I=1,2, \ldots, n$ contains only one fuzzy region (i.e., the whole interval). Membership functions for the $i$ th variable are defined as shown in Fig. 8.

Having defined membership functions, we need to generate fuzzy rules from given data pairs. We use if-then rules of the form IF $\boldsymbol{x}^{k}$ is in $H(\boldsymbol{y})$ THEN $\boldsymbol{z}=\boldsymbol{R}^{k}$, where $\boldsymbol{z}$ is the output of the rule. At the level of vector components, this means

$$
\begin{gathered}
\mathrm{IF} x_{1}^{k} \text { is in }\left[y_{1}, y_{1}+\lambda_{1}\right] \text { AND } x_{2}^{k} \text { is in }\left[y_{2}, y_{2}+\lambda_{2}\right] \text { AND } \ldots \\
\ldots \text { AND } x_{n}^{k} \text { is in }\left[y_{n}, y_{n}+\lambda_{n}\right] \text { THEN } z=R^{k}
\end{gathered}
$$




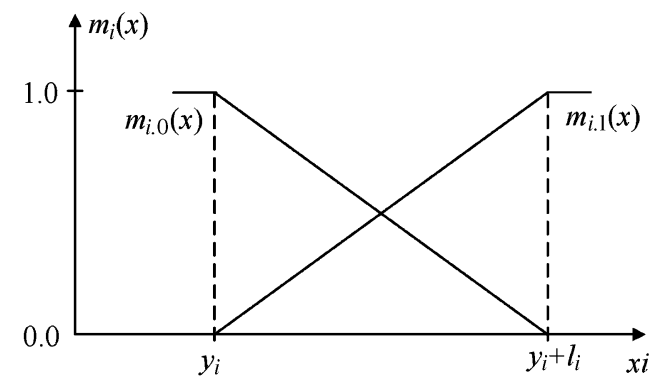

Fig. 8. Input interval $\left[y_{i}, y_{i}+\lambda_{i}\right]$ and the corresponding membership functions.

where $x_{i}^{k}, i=1, \ldots, n$ are components of vector $\boldsymbol{x}^{k}$. In our case, all $N$ rules are conflicting because they have the same IF part, but a different THEN part. However, each rule has a different set of associated membership functions. In particular, if $x_{j}^{k}=$ $y_{j}$, then the membership function associated with component $j$ of the $k$ th rule is $m_{j .0}$, otherwise it is $m_{j .1}$.

Each rule has a degree that is assigned in the following way. For the rule "IF $x_{1}^{k}$ is in $\left[y_{1}, y_{1}+\lambda_{1}\right]$ AND $x_{2}^{k}$ is in $\left[y_{2}, y_{2}+\lambda_{2}\right]$ AND $\ldots \ldots$ AND $x_{n}^{k}$ is in $\left[y_{n}, y_{n}+\lambda_{n}\right]$ THEN $\boldsymbol{z}=\boldsymbol{R}^{k,}$, the degree of this rule for any $\boldsymbol{x}=\left[\begin{array}{llll}x_{1} & x_{2} & \ldots & x_{n}\end{array}\right]^{T} \in H(\boldsymbol{y})$, denoted by $D_{k}(\boldsymbol{x})$, is defined as

$$
D_{k}(\boldsymbol{x})=\prod_{j=1}^{n} m_{j . e_{j}^{k}}\left(x_{j}\right)
$$

where $e_{j}^{k} \in\{0,1\}, j=1,2, \ldots, n$ are coefficients in the following expansion of $x_{k}: \boldsymbol{x}^{k}=$ $\left[\begin{array}{lll}y_{1}+e_{1}^{k} \lambda_{1} & y_{2}+e_{2}^{k} \lambda_{2} & y_{n}+e_{n}^{k} \lambda_{n}\end{array}\right]^{T}, k=1,2, \ldots, N$.

The output of our fuzzy system is determined using centroid defuzzification

$$
F(\boldsymbol{x})=\frac{\sum_{j=1}^{N} D_{k}(\boldsymbol{x}) \boldsymbol{R}^{k}}{\sum_{j=1}^{N} D_{k}(\boldsymbol{x})}
$$

which is a realization of an interpolated coarse model $\overline{\boldsymbol{R}}_{c}(1)$ and can be written as $\overline{\boldsymbol{R}}_{c}(\boldsymbol{x})=F\left(\boldsymbol{x}, X_{B}(s(\boldsymbol{x})), R_{B}(s(\boldsymbol{x}))\right)$ since $F$ is a function of both $X_{B}$ and $R_{B}$.

As mentioned before, in this paper, we only use triangular membership functions. This assures that $\overline{\boldsymbol{R}}_{c}$ is a continuous function over the whole domain of the coarse model (regardless of the continuity of the original coarse model $\boldsymbol{R}_{c}$ ). Other choices, e.g., z-shaped membership functions, would permit keeping first-order differentiability of the interpolated model, which may be important for some problems.

\section{Implementation Details}

In order to reduce the number of evaluations of the original coarse model $\boldsymbol{R}_{c}$, the interpolated model is implemented as a database of interpolating functions (4), which is updated if the coarse model needs to be evaluated. The coarse model evaluation process can be described by the following algorithm $(D B$ denotes the model database).

1. Get $\boldsymbol{x}$ (the point to evaluate the coarse model at)

2. If $s(\boldsymbol{x}) \in D B$, retrieve the interpolation function and return $\overline{\boldsymbol{R}}_{c}(\boldsymbol{x})=F\left(\boldsymbol{x}, X_{B}(s(\boldsymbol{x})), R_{B}(s(\boldsymbol{x}))\right)$
3. Generate the base set $X_{B}(s(\boldsymbol{x}))$

4. For each $\boldsymbol{z} \in X B(s(\boldsymbol{x}))$ calculate $\boldsymbol{R}_{c}(\boldsymbol{z})$; create $R_{B}(s(\boldsymbol{x}))$

5. Prepare fuzzy rules; prepare interpolating function $F$

6. Update $D B$ (save $s(\boldsymbol{x})$ and corresponding $F$ )

7. Return $\overline{\boldsymbol{R}}_{c}(\boldsymbol{x})=F\left(\boldsymbol{x}, X_{B}(s(\boldsymbol{x})), R_{B}(s(\boldsymbol{x}))\right)$

In other words, if $\boldsymbol{x}$ belongs to a hypercube for which the interpolating function is already set, the response of $\overline{\boldsymbol{R}}_{c}$ is obtained as the value of the interpolating function $F$ corresponding to this hypercube. Otherwise, $F$ must first be created (which requires setting up the base set, acquiring the original coarse model data, and calculating the necessary coefficients), then evaluated, and finally, stored in the database.

As mentioned before, the number of base points for each hypercube is $2^{n}$, i.e., the number grows exponentially with the number of design variables. However, in practice, many hypercubes considered during the optimization process are adjacent to each other. This means that many corner points are shared between hypercubes. Due to this, the actual average number of original coarse model evaluations per hypercube is smaller than $2^{n}$. We observed that, depending on the problem, the figure is $2^{n} / K$, where $K$ is typically from 2 to 4 .

It should also be noted that there is a tradeoff between the accuracy of the interpolated coarse model and its computational cost. On one hand, we want to take advantage of the accuracy of the original coarse model, as this would allow us to maintain the number of fine model evaluation as low during the space-mapping optimization process. On the other hand, we need to keep the interpolated coarse model fast; otherwise the benefits of using space-mapping optimization are lost due to the computational overhead related to parameter extraction and surrogate model optimization. Both model accuracy and speed depend on the user-defined grid $G$, and the grid size should be adjusted so that both the accuracy and computational cost of the interpolated coarse model are sufficient. This may be easily achieved if the number of design variables $n$ is small, such as two or three. For larger values of $n$, due to the exponential growth of the number of base points for each hypercube, the number of actual evaluations of the original coarse model may be too large and all the benefits of using our interpolation scheme may be lost. In practice, our method should not be used for $n>4$ unless the model is not highly nonlinear. Another method working regardless of the number of design variables will be described elsewhere.

\section{EXAMPLES}

As a first example, consider again the second-order tapped-line microstrip filter described in Section II. We optimized this filter again, using the interpolated model (4) based on the original coarse model $\boldsymbol{R}_{c 1}$ with grid size $\boldsymbol{\lambda}=\left[\begin{array}{ll}1 & 0.02\end{array}\right]^{T} \mathrm{~mm}$. Table II shows the optimization results (the optimized design is $[6.0650 .056]^{T}$ ). It is seen that space-mapping optimization with the interpolated model gives the same final specification error as optimization with models $\boldsymbol{R}_{c 1}$ and $\boldsymbol{R}_{c 2}$ (cf. Section II), but with substantially smaller computational cost.

The reduction of the total optimization time in comparison to optimization with $\boldsymbol{R}_{c 1}\left(\boldsymbol{R}_{c 2}\right)$ is $69 \%$ (56\%). Most of the savings arise from using the interpolated coarse model. This resulted in 
TABLE II

SPACE-MAPPING OPTIMIZATION RESUltS FOR THE SECOND-ORDER TAPPED-LINE MICROSTRIP FILTER (WITH INTERPOLATED COARSE MODEL)

\begin{tabular}{|c|c|c|c|c|c|}
\hline $\begin{array}{l}\text { Coarse } \\
\text { Model }\end{array}$ & $\begin{array}{l}\text { Final } \\
\text { Spec. } \\
\text { Error } \\
{[\mathrm{dB}]} \\
\end{array}$ & $\begin{array}{l}\text { Number of } \\
\text { Fine Model } \\
\text { Evaluations }\end{array}$ & $\begin{array}{c}\text { Total } \\
\text { Optimization } \\
\text { Time }\end{array}$ & $\begin{array}{c}\text { Total } \\
\text { Fine Model } \\
\text { Evaluation } \\
\text { Time } \\
\end{array}$ & $\begin{array}{c}\text { Total Parameter } \\
\text { Extraction } \\
\text { and Surrogate } \\
\text { Optimization Time }\end{array}$ \\
\hline$\overline{\boldsymbol{R}}_{c}$ & -0.65 & 2 & $11.1 \mathrm{~min}$ & $9.7 \mathrm{~min}(87 \%)$ & $1.4 \min (13 \%)$ \\
\hline
\end{tabular}

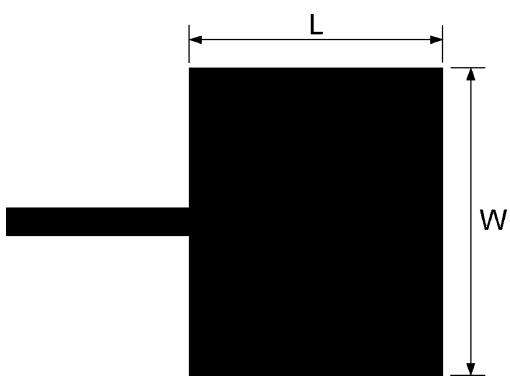

Fig. 9. Geometry of the patch antenna.

a reduction of the total number of evaluations of $\boldsymbol{R}_{c 1}$ to 8 (versus 115 when directly using $\boldsymbol{R}_{c 1}$ in space-mapping optimization).

Consider the patch antenna shown in Fig. 9. This antenna is printed on a substrate with relative dielectric constant $\varepsilon_{r}=2.32$ and height $h=1.59 \mathrm{~mm}$. The design parameters are the patch length and width, i.e., $\boldsymbol{x}=[L W]^{T}$. The objective is to obtain $50-\Omega$ input impedance at $2 \mathrm{GHz}$. The fine model $\boldsymbol{R}_{f}$ is simulated in FEKO [32]. The number of meshes for the fine model is 1024, which ensures mesh convergence for the structure. Simulation time for the fine model is $34 \mathrm{~s}$.

We consider two coarse models. Model $\boldsymbol{R}_{c}$ is the structure in Fig. 9 also simulated in FEKO, however, the number of meshes is only 100. Simulation time for model $\boldsymbol{R}_{c}$ is $0.45 \mathrm{~s}$. Model $\overline{\boldsymbol{R}}_{c}$ is an interpolated model (4) based on the original coarse model $\boldsymbol{R}_{c}$ with grid size $\boldsymbol{\lambda}=\left[\begin{array}{ll}1 & 2\end{array}\right]^{T} \mathrm{~mm}$. The number of meshes for $\boldsymbol{R}_{f}$ and $\boldsymbol{R}_{c}$ are measured at the optimal solution of $\overline{\boldsymbol{R}}_{c}$, which is $\boldsymbol{x}^{(0)}=[46.49100 .89]^{T} \mathrm{~mm}$. The fine model response at $\boldsymbol{x}^{(0)}$ is $38.8 \Omega$.

For this problem, we used the same space-mapping surrogate model as described in Section II. We perform space-mapping optimization twice: using model $\boldsymbol{R}_{c}$ and then using model $\overline{\boldsymbol{R}}_{c}$. In both cases, we use the same starting point $\boldsymbol{x}^{(0)}$.

Table III shows the optimization results. As we see, the final value of the input impedance is similar in both cases (the corresponding final designs are [46.69 100.99] $]^{T}$ for $\overline{\boldsymbol{R}}_{c}$ and [46.64 99.94] ${ }^{T}$ for $\boldsymbol{R}_{c}$ ), although the accuracy is better for $\overline{\boldsymbol{R}}_{c}$ than for $\boldsymbol{R}_{c}$ (a specification error of $0.05 \Omega$ for $\overline{\boldsymbol{R}}_{c}$ versus $0.09 \Omega$ for $\boldsymbol{R}_{c}$ ). The computational cost of space-mapping optimization is also more than two times smaller for $\overline{\boldsymbol{R}}_{c}$ than for $\boldsymbol{R}_{c}$, which is because the total number of evaluations of the original coarse model has been reduced from more than 500 (when directly using $\boldsymbol{R}_{c}$ in space-mapping optimization) to 29 (when using $\boldsymbol{R}_{c}$ ).

Consider the microstrip notch filter with mitered bends [37] shown in Fig. 10. The design parameters are $x=$ $\left[\begin{array}{lll}L_{c} & L_{o} & S_{g}\end{array}\right]^{T}$ mil. Other parameters are $W=30 \mathrm{mil}$,
TABLE III

Space-MAPPing Optimization Results For the PATCh ANTENNA

\begin{tabular}{|c|c|c|c|c|c|}
\hline $\begin{array}{l}\text { Coarse } \\
\text { Model }\end{array}$ & $\begin{array}{c}\text { Final } \\
Z_{\text {in }} \\
{[\Omega]}\end{array}$ & $\begin{array}{l}\text { Number of } \\
\text { Fine Model } \\
\text { Evaluations }\end{array}$ & $\begin{array}{c}\text { Total } \\
\text { Optimization } \\
\text { Time }\end{array}$ & $\begin{array}{c}\text { Total } \\
\text { Fine Model } \\
\text { Evaluation } \\
\text { Time } \\
\end{array}$ & $\begin{array}{c}\text { Total Parameter } \\
\text { Extraction } \\
\text { and Surrogate } \\
\text { Optimization Time }\end{array}$ \\
\hline $\boldsymbol{R}_{C}$ & 50.09 & 5 & $414 \mathrm{~s}$ & $170 \mathrm{~s}(41 \%)$ & $244 \mathrm{~s}(59 \%)$ \\
\hline$\overline{\boldsymbol{R}}_{c}$ & 49.95 & 5 & $183 \mathrm{~s}$ & $170 \mathrm{~s}(93 \%)$ & $13 \mathrm{~s}(7 \%)$ \\
\hline
\end{tabular}

* Includes fine model evaluation at the starting point

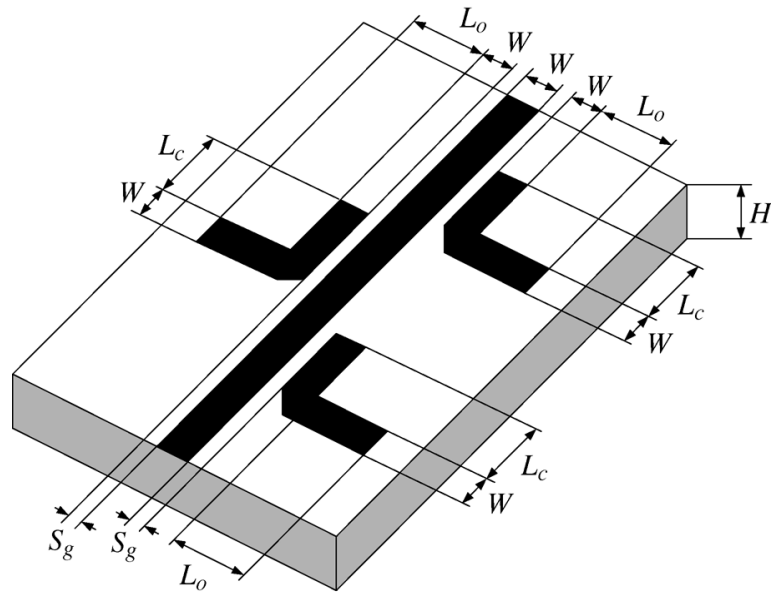

Fig. 10. Microstrip notch filter with mitered bends [37].

$H=10$ mil, and $\varepsilon_{r}=2.2$ (loss tangent 0.0009). The fine $\boldsymbol{R}_{f}$ model is simulated in Sonnet $\boldsymbol{e m}$ [38] with a fine grid of $0.5 \mathrm{mil} \times 0.5 \mathrm{mil}$. The simulation time for $\boldsymbol{R}_{f}$ is $1 \mathrm{~h}$ and $34 \mathrm{~min}$ (12 points per frequency sweep). The design specifications are

$$
\begin{aligned}
& \left|S_{21}\right| \geq 0.95 \text { for } 12.7 \mathrm{GHz} \leq \omega \leq 13.0 \mathrm{GHz} \\
& \left|S_{21}\right| \leq 0.05 \text { for } 13.19 \mathrm{GHz} \leq \omega \leq 13.21 \mathrm{GHz} \\
& \left|S_{21}\right| \geq 0.95 \text { for } 13.4 \mathrm{GHz} \leq \omega \leq 13.7 \mathrm{GHz} .
\end{aligned}
$$

We consider the original coarse model $\boldsymbol{R}_{c}$, which is also simulated in Sonnet $\boldsymbol{e m}$, however, with a coarse grid of $5 \mathrm{mil} \times 1 \mathrm{mil}$. The simulation time for $\boldsymbol{R}_{c}$ is $65 \mathrm{~s}$. Obviously, $\boldsymbol{R}_{c}$ cannot be directly used in the optimization process because it is available only on a coarse grid. Instead, we use model $\overline{\boldsymbol{R}}_{c}$, which is an interpolated model (4) based on $\boldsymbol{R}_{c}$ with grid size $\boldsymbol{\lambda}=$ $\left[\begin{array}{lll}5 & 1 & 1\end{array}\right]^{T}$ mil. The optimal solution of this model is $\boldsymbol{x}_{1}^{(0)}=$ $\left[\begin{array}{llll}145 & 158 & 8\end{array}\right]^{T}$ mil. Fig. 11 shows the fine and coarse model responses at $\boldsymbol{x}^{(0)}$.

We also use another coarse model, i.e., $\boldsymbol{R}_{c 1}$, which is the circuit model implemented in Agilent ADS [33] and shown in Fig. 12. The evaluation time for $\boldsymbol{R}_{c 1}$ is approximately $1.5 \mathrm{~s}$. Model $\boldsymbol{R}_{c 1}$ has its substrate permittivity tuned to $\varepsilon_{r}=1.46$, which allows us to shift the center frequency of its response to $13.2 \mathrm{GHz}$ at $\boldsymbol{x}_{1}^{(0)}$. Without tuning, the center frequency of $\boldsymbol{R}_{c 1}$ is approximately $11.12 \mathrm{GHz}$. This causes severe misalignment between the fine model and $\boldsymbol{R}_{c 1}$ and makes it unsuitable for space-mapping optimization. Fig. 13 shows the responses of $\boldsymbol{R}_{c 1}$ at $\boldsymbol{x}_{1}^{(0)}$ before and after the tuning of $\varepsilon_{r}$.

To solve our problem, we used the same space-mapping surrogate model described in Section II. We perform space-mapping optimization twice: using model $\overline{\boldsymbol{R}}_{c}$ with 


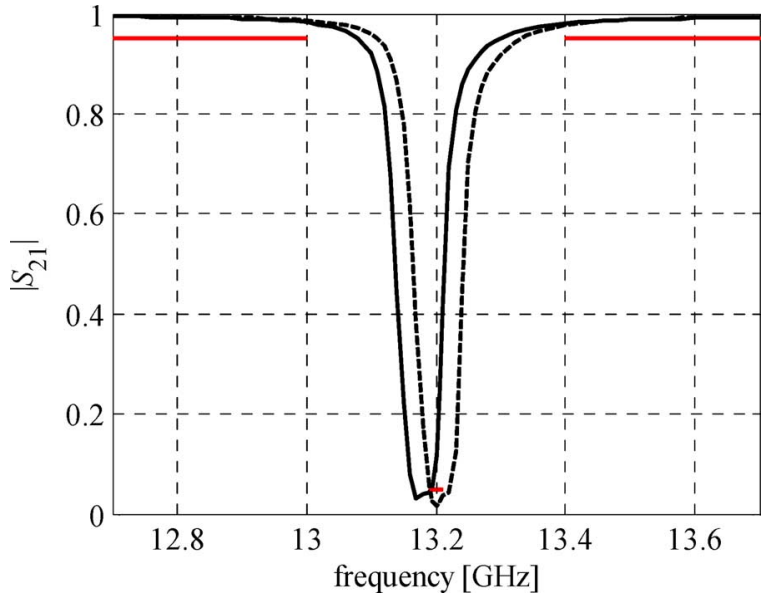

Fig. 11. Microstrip notch filter: initial fine model response (solid line) and coarse model $\overline{\boldsymbol{R}}_{c}$ response (dashed line) at $\boldsymbol{x}_{1}^{(0)}$.

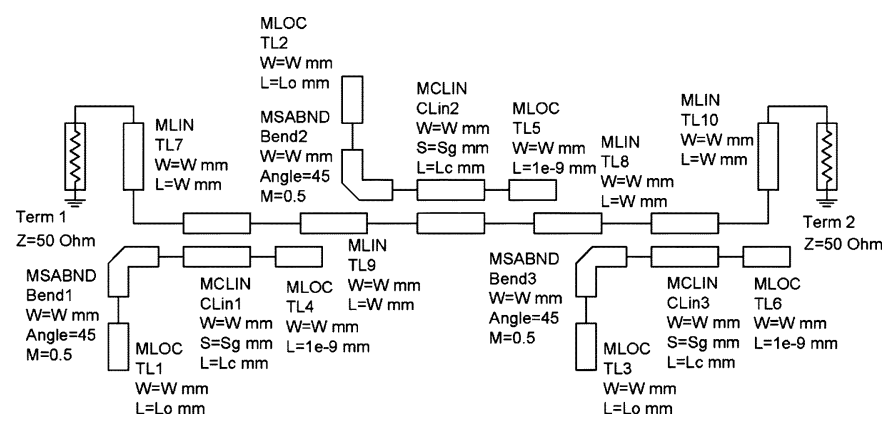

Fig. 12. Coarse model $\boldsymbol{R}_{c 1}$ of the notch filter (Agilent ADS).

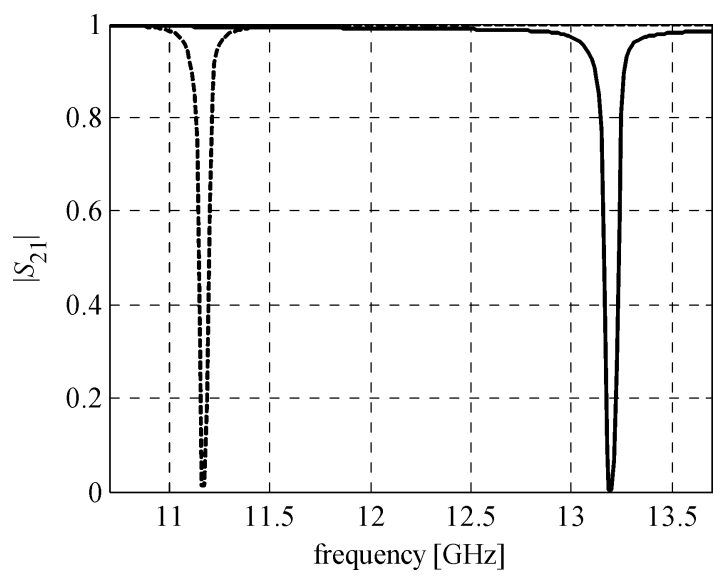

Fig. 13. Microstrip notch filter: response of the coarse model $\boldsymbol{R}_{c 1}$ at $\boldsymbol{x}_{1}^{(0)}$ without tuning of $\varepsilon_{r}$ (dashed line) and with $\varepsilon_{r}$ tuned to 1.46 (solid line).

starting point $\boldsymbol{x}_{1}^{(0)}$ and then using model $\boldsymbol{R}_{c 1}$ with starting point $\boldsymbol{x}_{2}^{(0)}=\left[\begin{array}{lll}145 & 158 & 9\end{array}\right]^{T}$ (the optimal solution of the (tuned) $\boldsymbol{R}_{c 1}$ ).

Table IV shows the optimization results. As we can see, the final solutions (the responses shown in Fig. 14) satisfy the design specification in both cases (the corresponding final designs are $\left[\begin{array}{llll}145 & 157.5 & 8\end{array}\right]^{T}$ for $\overline{\boldsymbol{R}}_{c}$ and $\left[\begin{array}{llll}144.5 & 158 & 7\end{array}\right]^{T}$ for $\boldsymbol{R}_{c 1}$ ). The computational cost of space-mapping optimization, however, is
TABLE IV

SPACE-MAPPING OPtIMIZATION RESULTS FOR THE MicRostrip NOTCH FILTER

\begin{tabular}{|c|c|c|c|c|c|}
\hline $\begin{array}{l}\text { Coarse } \\
\text { Model }\end{array}$ & $\begin{array}{l}\text { Final } \\
\text { Spec. } \\
\text { Error }\end{array}$ & $\begin{array}{l}\text { Number of } \\
\text { Fine Model } \\
\text { Evaluations }\end{array}$ & $\begin{array}{c}\text { Total } \\
\text { Optimization } \\
\text { Time }\end{array}$ & $\begin{array}{c}\text { Total } \\
\text { Fine Model } \\
\text { Evaluation } \\
\text { Time }\end{array}$ & $\begin{array}{c}\text { Total Parameter } \\
\text { Extraction } \\
\text { and Surrogate } \\
\text { Optimization } \\
\text { Time } \\
\end{array}$ \\
\hline$\overline{\boldsymbol{R}}_{c}$ & -0.006 & 2 & $3 \mathrm{~h} 36 \mathrm{~min}$ & $3 \mathrm{~h} 8 \min (87 \%)$ & $13 \%)$ \\
\hline $\boldsymbol{R}_{C 1}$ & -0.02 & 6 & $9 \mathrm{~h} 40 \mathrm{~min}$ & $9 \mathrm{~h} 24 \mathrm{~min}(97 \%)$ & $16 \mathrm{~min}(3 \%)$ \\
\hline
\end{tabular}

* Includes fine model evaluation at the starting point

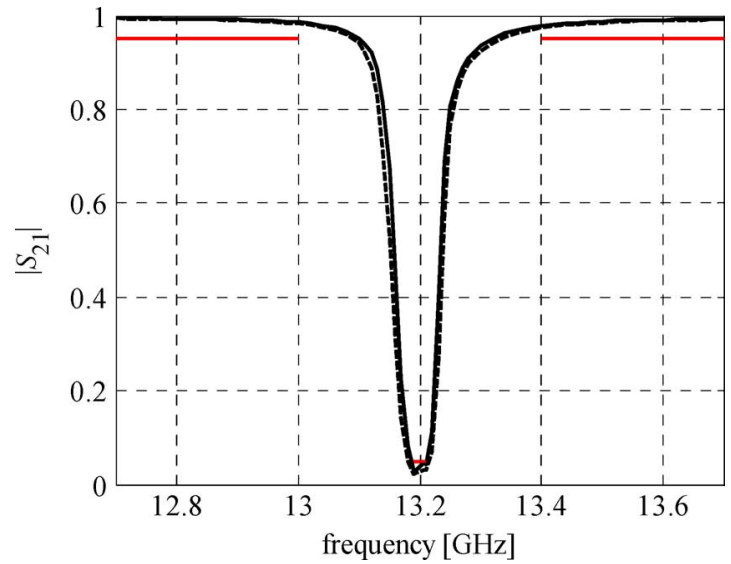

Fig. 14. Microstrip notch filter: final fine model response at the solution obtained with space mapping using $\overline{\boldsymbol{R}}_{c}$ model (solid line) and $\boldsymbol{R}_{c 1}$ model (dashed line).

substantially smaller for $\overline{\boldsymbol{R}}_{c}$ than for $\boldsymbol{R}_{c 1}$. This is because $\overline{\boldsymbol{R}}_{c}$ is more accurate than $\boldsymbol{R}_{c 1}$.

Note that although the evaluation time for the original coarse model $\boldsymbol{R}_{c}$ is $65 \mathrm{~s}$, the total time required for parameter extraction and surrogate optimization is only $28 \mathrm{~min}$. This is because our interpolated model only required 26 evaluations of the original coarse model.

\section{CONCLUSION}

An interpolation technique for creating coarse models suitable for space-mapping optimization has been presented. Our technique allow us to build models that are tradeoffs between accuracy and computational cost. As a result, we are able to reduce the computational cost of space-mapping optimization by decreasing the number of fine model evaluations necessary to obtain satisfactory solutions (because of good coarse model accuracy), as well as by reducing the total cost of solving the parameter extraction and surrogate optimization sub-problems (because the interpolated coarse model is faster than the original coarse model). Examples demonstrate the robustness of our approach.

\section{ACKNOWLEDGMENT}

The authors thank Sonnet Software Inc., Syracuse, NY, for em, and Agilent Technologies, Santa Rosa, CA, for making ADS available. The authors acknowledge discussions with 
J. E. Rayas-Sánchez, Instituto Tecnológico y de Estudios Superiores de Occidente (ITESO), Guadalajara, Mexico, and his help on the notch filter example.

\section{REFERENCES}

[1] J. W. Bandler, R. M. Biernacki, S. H. Chen, P. A. Grobelny, and R. H. Hemmers, "Space mapping technique for electromagnetic optimization," IEEE Trans. Microw. Theory Tech., vol. 4, no. 12, pp. 536-544, Dec. 1994

[2] J. W. Bandler, Q. S. Cheng, N. K. Nikolova, and M. A. Ismail, "Implicit space mapping optimization exploiting preassigned parameters," IEEE Trans. Microw. Theory Tech., vol. 52, no. 1, pp. 378-385, Jan. 2004.

[3] J. W. Bandler, Q. S. Cheng, D. H. Gebre-Mariam, K. Madsen, F. Pedersen, and J. Søndergaard, "EM-based surrogate modeling and design exploiting implicit, frequency and output space mappings," in IEEE MTT-S Int. Microw. Symp. Dig., Philadelphia, PA, Jun. 2003, pp. 1003-1006.

[4] J. W. Bandler, Q. S. Cheng, S. A. Dakroury, A. S. Mohamed, M. H. Bakr, K. Madsen, and J. Søndergaard, "Space mapping: The state of the art," IEEE Trans. Microw. Theory Tech., vol. 52, no. 1, pp. 337-361, Jan. 2004.

[5] D. Echeverria and P. W. Hemker, "Space mapping and defect correction," Int. Math. J. Comput. Methods Appl. Math., vol. 5, no. 2, pp. 107-136, 2005.

[6] N. M. Alexandrov and R. M. Lewis, "An overview of first-order model management for engineering optimization," Optimization Eng., vol. 2, no. 4, pp. 413-430, Dec. 2001.

[7] A. J. Booker, J. E. Dennis, Jr., P. D. Frank, D. B. Serafini, V. Torczon, and M. W. Trosset, "A rigorous framework for optimization of expensive functions by surrogates," Structural Optimization, vol. 17, no. 1, pp. 1-13, Feb. 1999.

[8] J. E. Dennis and V. Torczon, "Managing approximation models in optimization," in Multidisciplinary Design Optimization, N. M. Alexandrov and M. Y. Hussaini, Eds. Philadelphia, PA: SIAM, 1997, pp. $330-374$.

[9] S. J. Leary, A. Bhaskar, and A. J. Keane, "A knowledge-based approach to response surface modeling in multifidelity optimization," Global Optimization, vol. 26, no. 3, pp. 297-319, Jul. 2003.

[10] S. E. Gano, J. E. Renaud, and B. Sanders, "Variable fidelity optimization using a Kriging based scaling function," presented at the 10th AIAA/ISSMO Multidisciplinary Anal. Optimization Conf., Albany, NY, 2004, Paper AIAA-2004-4460.

[11] T. W. Simpson, J. Peplinski, P. N. Koch, and J. K. Allen, "Metamodels for computer-based engineering design: Survey and recommendations," Eng. Comput., vol. 17, no. 2, pp. 129-150, Jul. 2001.

[12] N. V. Queipo, R. T. Haftka, W. Shyy, T. Goel, R. Vaidynathan, and P. K. Tucker, "Surrogate-based analysis and optimization," Progress Aerosp. Sci., vol. 41, no. 1, pp. 1-28, Jan. 2005.

[13] M. A. Ismail, D. Smith, A. Panariello, Y. Wang, and M. Yu, "EMbased design of large-scale dielectric-resonator filters and multiplexers by space mapping," IEEE Trans. Microw. Theory Tech., vol. 52, no. 1, pp. 386-392, Jan. 2004.

[14] K.-L. Wu, Y.-J. Zhao, J. Wang, and M. K. K. Cheng, "An effective dynamic coarse model for optimization design of LTCC RF circuits with aggressive space mapping," IEEE Trans. Microw. Theory Tech., vol. 52, no. 1, pp. 393-402, Jan. 2004.

[15] S. Amari, C. LeDrew, and W. Menzel, "Space-mapping optimization of planar coupled-resonator microwave filters," IEEE Trans. Microw. Theory Tech., vol. 54, no. 5, pp. 2153-2159, May 2006.

[16] M. Dorica and D. D. Giannacopoulos, "Response surface space mapping for electromagnetic optimization," IEEE Trans. Magn., vol. 42, no. 4, pp. 1123-1126, Apr. 2006.

[17] J. Zhu, J. W. Bandler, N. K. Nikolova, and S. Koziel, "Antenna optimization through space mapping," IEEE Trans. Antennas Propag., vol. 55, no. 3, pp. 651-658, Mar. 2007.

[18] S. J. Leary, A. Bhaskar, and A. J. Keane, "A constraint mapping approach to the structural optimization of an expensive model using surrogates," Optimization Eng., vol. 2, no. 4, pp. 385-398, Dec. 2001 .

[19] M. Redhe and L. Nilsson, "Using space mapping and surrogate models to optimize vehicle crashworthiness design," presented at the 9th AIAA/ISSMO Multidisciplinary Anal. Optimization Symp., Atlanta, GA, Sep. 2002, Paper AIAA-2002-5536.
[20] H.-S. Choi, D. H. Kim, I. H. Park, and S. Y. Hahn, "A new design technique of magnetic systems using space mapping algorithm," IEEE Trans. Magn., vol. 37, no. 5, pp. 3627-3630, Sep. 2001.

[21] S. Koziel, J. W. Bandler, and K. Madsen, "A space mapping framework for engineering optimization: Theory and implementation," IEEE Trans. Microw. Theory Tech., vol. 54, no. 10, pp. 3721-3730, Oct. 2006.

[22] S. Koziel and J. W. Bandler, "Space-mapping optimization with adaptive surrogate model," IEEE Trans. Microw. Theory Tech., vol. 55, no. 3, pp. 541-547, Mar. 2007

[23] S. Koziel, J. W. Bandler, A. S. Mohamed, and K. Madsen, "Enhanced surrogate models for statistical design exploiting space mapping technology," in IEEE MTT-S Int. Microw. Symp. Dig., Long Beach, CA, Jun. 2005, pp. 1609-1612.

[24] S. Koziel, J. W. Bandler, and K. Madsen, "Theoretical justification of space-mapping-based modeling utilizing a data base and on-demand parameter extraction," IEEE Trans. Microw. Theory Tech., vol. 54, no. 12, pp. 4316-4322, Dec. 2006

[25] V. K. Devabhaktuni, B. Chattaraj, M. C. E. Yagoub, and Q.-J. Zhang, "Advanced microwave modeling framework exploiting automatic model generation, knowledge neural networks, and space mapping," IEEE Trans. Microw. Theory Tech., vol. 51, no. 7, pp. 1822-1833, Jul. 2003.

[26] J. E. Rayas-Sánchez, "EM-based optimization of microwave circuits using artificial neural networks: The state-of-the-art," IEEE Trans. Microw. Theory Tech., vol. 52, no. 1, pp. 420-435, Jan. 2004.

[27] J. E. Rayas-Sánchez, F. Lara-Rojo, and E. Martinez-Guerrero, "A linear inverse space-mapping (LISM) algorithm to design linear and nonlinear RF and microwave circuits," IEEE Trans. Microw. Theory Tech., vol. 53, no. 3, pp. 960-968, Mar. 2005.

[28] L. Zhang, J. Xu, M. C. E. Yagoub, R. Ding, and Q.-J. Zhang, "Efficient analytical formulation and sensitivity analysis of neuro-space mapping for nonlinear microwave device modeling," IEEE Trans. Microw. Theory Tech., vol. 53, no. 9, pp. 2752-2767, Sep. 2005.

[29] S. Koziel, J. W. Bandler, and K. Madsen, "Towards a rigorous formulation of the space mapping technique for engineering design," in Proc. Int Circuits Syst. Symp., Kobe, Japan, May 2005, pp. 5605-5608.

[30] K. Madsen and J. Søndergaard, "Convergence of hybrid space mapping algorithms," Optimization Eng., vol. 5, no. 2, pp. 145-156, Jun. 2004.

[31] A. Manchec, C. Quendo, J.-F. Favennec, E. Rius, and C. Person, "Synthesis of capacitive-coupled dual-behavior resonator (CCDBR) filters," IEEE Trans. Microw. Theory Tech., vol. 54, no. 6, pp. 2346-2355, Jun. 2006.

[32] “FEKO User's Manual, Suite 4.2,” EM Softw. Syst. S.A. (Pty) Ltd., Stellenbosch, South Africa, 2004. [Online]. Available: http://www.feko.info

[33] Agilent ADS. ver. 2003C, Agilent Technol., Santa Rosa, CA, 2003.

[34] V. Miraftab and R. R. Mansour, "Computer-aided tuning of microwave filters using fuzzy logic," IEEE Trans. Microw. Theory Tech., vol. 50, no. 12 , pp. 2781-2788, Dec. 2002.

[35] V. Miraftab and R. R. Mansour, "A robust fuzzy-logic technique for computer-aided diagnosis of microwave filters," IEEE Trans. Microw. Theory Tech., vol. 52, no. 1, pp. 450-456, Jan. 2004

[36] L.-X. Wang and J. M. Mendel, "Generating fuzzy rules by learning from examples," IEEE Trans. Syst., Man, Cybern., vol. 22, no. 6, pp. 1414-1427, Nov./Dec. 1992.

[37] J. E. Rayas-Sánchez and V. Gutiérrez-Ayala, "EM-based Monte Carlo analysis and yield prediction of microwave circuits using linear-input neural-output space mapping," IEEE Trans. Microw. Theory Tech., vol. 54, no. 12 , pp. $4528-4537$, Dec. 2006.

[38] $\boldsymbol{e m}$. ver. 10.53, Sonnet Softw., North Syracuse, NY, 2006.

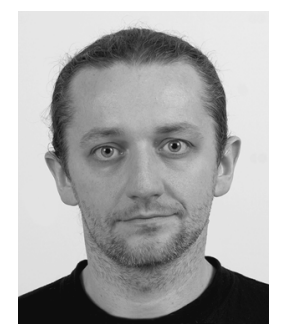

Slawomir Koziel (M'03-SM'07) received the M.Sc. and $\mathrm{Ph} . \mathrm{D}$. degrees in electronic engineering from Gdansk University of Technology, Gdansk, Poland, in 1995 and 2000, respectively, and the M.Sc. degrees in theoretical physics and in mathematics and $\mathrm{Ph} . \mathrm{D}$. degree in mathematics, from the University of Gdansk, Gdansk, Poland, in 2000, 2002, and 2003 , respectively.

$\mathrm{He}$ is currently a Research Associate with the Department of Electrical and Computer Engineering, McMaster University, Hamilton, ON, Canada. He has authored or coauthored over 100 papers. His research interests include space mapping, circuit theory, analog signal processing, evolutionary computation, and numerical analysis. 


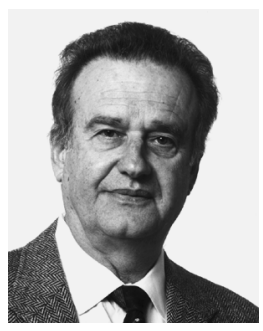

John W. Bandler (S'66-M'66-SM'74-F'78LF'06) studied at Imperial College. He received the B.Sc. (Eng.), Ph.D., and D.Sc. (Eng.) degrees from the University of London, London, U.K., in 1963, 1967, and 1976, respectively.

In 1969, he joined McMaster University, Hamilton, ON, Canada, where he is currently Professor Emeritus. He was President of Optimization Systems Associates Inc., which he founded in 1983, until November 20,1997, the date of acquisition by the Hewlett-Packard Company. He is President of Bandler Corporation, Dundas, ON, Canada, which he founded in 1997.

Dr. Bandler is a Fellow of several societies including the Royal Society of Canada. He was the recipient of the 2004 IEEE Microwave Theory and Techniques Society (IEEE MTT-S) Microwave Application Award. 